\title{
Pacific
}

Journal of

Mathematics

\section{A QUANTITATIVE ESTIMATE FOR QUASIINTEGRAL POINTS IN ORBITS}

LiAng-Chung Hsia AND JosePh H. SILVERMAN 


\title{
A QUANTITATIVE ESTIMATE FOR QUASIINTEGRAL POINTS IN ORBITS
}

\author{
LIANG-CHUNG HSIA AND JOSEPH H. SILVERMAN
}

Let $\varphi(z) \in K(z)$ be a rational function of degree $d \geq 2$ defined over a number field whose second iterate $\varphi^{2}(z)$ is not a polynomial, and let $\alpha \in K$. The second author previously proved that the forward orbit $O_{\varphi}(\alpha)$ contains only finitely many quasi- $S$-integral points. We give an explicit upper bound for the number of such points.

\section{Introduction}

Let $K / \mathbb{Q}$ be a number field, let $S$ be a finite set of places of $K$, and let $1 \geq \varepsilon>0$. An element $x \in K$ is said to be quasi- $(S, \varepsilon)$-integral if

$$
\sum_{v \in S} \frac{\left[K_{v}: \mathbb{Q}_{v}\right]}{[K: \mathbb{Q}]} \log ^{+}|x|_{v} \geq \varepsilon h(x) .
$$

We observe that $x$ is in the ring of $S$-integers of $K$ if and only if it is quasi- $(S, 1)$ integral, in which case (1) is an equality by definition of the height.

Let $\varphi(z) \in K(z)$ be a rational function of degree $d \geq 2$, let $\alpha \in K$ be a point, and let

$$
\mathrm{O}_{\varphi}(\alpha)=\left\{\alpha, \varphi(\alpha), \varphi^{2}(\alpha), \ldots\right\}
$$

denote the forward orbit of $\alpha$ under iteration of $\varphi$. Silverman [1993] proved that if $\varphi^{2}(z)$ is not a polynomial, then the orbit $\mathbb{O}_{\varphi}(\alpha)$ contains only finitely many quasi$(S, \varepsilon)$-integral points. More generally, if $\# \mathscr{O}_{\varphi}(\alpha)=\infty$ and if $\beta$ is not an exceptional point for $\varphi$, then there are only finitely many $n \geq 1$ such that

$$
\frac{1}{\varphi^{n}(\alpha)-\beta}
$$

is quasi- $(S, \varepsilon)$-integral. In this note we give an upper bound for the number of such $n$, making explicit the dependence on $S, \varphi, \alpha$, and $\beta$. More precisely, we

Hsia is supported by NSC-97-2918-I-008-005 and NSC-96-2115-008-012-MY3. Silverman is supported by NSF DMS-0650017 and DMS-0854755.

MSC2010: primary 37P15; secondary 11B37, 11G99, 14G99.

Keywords: arithmetic dynamics, integral points. 
prove that the number of elements in the set

$$
\left\{n \geq 0:\left(\varphi^{n}(\alpha)-\beta\right)^{-1} \text { is quasi- }(S, \varepsilon) \text {-integral }\right\}
$$

is smaller than

$$
4^{\# S} \gamma+\log _{d}^{+}\left(\frac{h(\varphi)+\hat{h}_{\varphi}(\beta)}{\hat{h}_{\varphi}(\alpha)}\right),
$$

where $\gamma$ depends only on $d, \varepsilon$, and $[K: \mathbb{Q}]$. (See Section 2 for the definitions of the height $h(\varphi)$ of the map $\varphi$ and the canonical height $\hat{h}_{\varphi}$.) Our main result, Theorem 11 in Section 5, is a strengthened version of this statement.

The specific form of the upper bound in (3) is interesting, especially the dependence on the wandering point $\alpha$ and the target point $\beta$. For example, if $\hat{h}_{\varphi}(\alpha)$ is sufficiently large (depending on $\beta$ and $\varphi$ ), then the bound is independent of $\alpha, \beta$, and $\varphi$. It is also interesting to ask whether it is possible, for a given $\varphi$ and $\alpha$, to make the set (2) arbitrarily large by varying $\beta$. We discuss this question further in Remark 14.

We briefly describe the organization of the paper. We start in Section 1 by setting notation and proving an elementary estimate for the chordal metric. Section 2 is devoted to height functions, both the canonical height associated to a rational map and various results relating heights and polynomials. In Section 3, we prove a uniform version of the inverse function theorem for rational maps of degree $d$. Section 4 states an estimate for the ramification of the iterate of a rational function, taken from [Silverman 1993; 2007], and a quantitative version of Roth's theorem, taken from [Silverman 1987b]. In Section 5 we combine the preliminary material to prove our main theorem. Finally, in Section 6, we use the main theorem to give an explicit upper bound for the number of $S$-integral points in an orbit.

Remark 1. Silverman's paper [1993] on finiteness of quasi- $S$-integral points in orbits has been used by Patrick Ingram and Silverman [2009] to prove a dynamical version of the classical Bang-Zsigmondy theorem on primitive divisors [Bang 1886; Zsigmondy 1892]. It has also been used by Felipe Voloch and Silverman [2009] to prove a local-global criterion for dynamics on $\mathbb{P}^{1}$. The quantitative results proved here should enable one to prove quantitative versions of the papers with Ingram and Voloch, but we have not included these applications in this paper in order to keep it to a manageable length.

Remark 2. Quantitative estimates similar to those in this paper have been proved for the number of integral points on elliptic curves and on certain other types of curves. See for example [Gross and Silverman 1995] and [Silverman 1987b]. 


\section{Preliminary material and notation}

We set the following notation:

$K \quad$ a number field.

$M_{K} \quad$ the set of places of $K$.

$M_{K}^{\infty} \quad$ the set of archimedean (infinite) places of $K$.

$M_{K}^{0} \quad$ the set of nonarchimedean (finite) places of $K$.

$\log ^{+}(x) \quad$ the maximum of $\log (x)$ and 0 . We write $\log _{d}^{+}$for $\log$ base $d$.

For each $v \in M_{K}$, we let $|\cdot|_{v}$ denote the corresponding normalized absolute value on $K$ whose restriction to $\mathbb{Q}$ gives the usual $v$-adic absolute value on $\mathbb{Q}$. That is, if $v \in M_{K}^{\infty}$, then $|x|_{v}$ is the usual archimedean absolute value, and if $v \in M_{K}^{0}$, then $|x|_{v}=|x|_{p}$ is the usual $p$-adic absolute value for a unique prime $p$. We also write $K_{v}$ for the completion of $K$ with respect to $|\cdot|_{v}$, and we let $\mathbb{C}_{v}$ denote the completion of an algebraic closure of $K_{v}$.

For each $v \in M_{K}$, we let $\rho_{v}$ denote the chordal metric defined on $\mathbb{P}^{1}\left(\mathbb{C}_{v}\right)$, where we recall that for $\left[x_{1}, y_{1}\right],\left[x_{2}, y_{2}\right] \in \mathbb{P}^{1}\left(\mathbb{C}_{v}\right)$,

$$
\rho_{v}\left(\left[x_{1}, y_{1}\right],\left[x_{2}, y_{2}\right]\right)= \begin{cases}\frac{\left|x_{1} y_{2}-x_{2} y_{1}\right|_{v}}{\sqrt{\left|x_{1}\right|_{v}^{2}+\left|y_{1}\right|_{v}^{2}} \sqrt{\left|x_{2}\right|_{v}^{2}+\left|y_{2}\right|_{v}^{2}}} & \text { if } v \in M_{K}^{\infty}, \\ \frac{\left|x_{1} y_{2}-x_{2} y_{1}\right|_{v}}{\max \left\{\left|x_{1}\right|_{v},\left|y_{1}\right|_{v}\right\} \max \left\{\left|x_{2}\right|_{v},\left|y_{2}\right|_{v}\right\}} & \text { if } v \in M_{K}^{0} .\end{cases}
$$

In this paper, we use the logarithmic version of the chordal metric to measure the distance between points in $\mathbb{P}^{1}\left(\mathbb{C}_{v}\right)$.

Definition. The logarithmic chordal metric function

$$
\lambda_{v}: \mathbb{P}^{1}\left(\mathbb{C}_{v}\right) \times \mathbb{P}^{1}\left(\mathbb{C}_{v}\right) \rightarrow \mathbb{R} \cup\{\infty\}
$$

is defined by

$$
\lambda_{v}\left(\left[x_{1}, y_{1}\right],\left[x_{2}, y_{2}\right]\right)=-\log \rho_{v}\left(\left[x_{1}, y_{1}\right],\left[x_{2}, y_{2}\right]\right) .
$$

Note that $\lambda_{v}(P, Q) \geq 0$ for all $P, Q \in \mathbb{P}^{1}\left(\mathbb{C}_{v}\right)$, and that two points $P, Q \in \mathbb{P}^{1}\left(\mathbb{C}_{v}\right)$ are close if and only if $\lambda_{v}(P, Q)$ is large. We also note that $\lambda_{v}$ is a particular choice of an arithmetic distance function as defined in [Silverman 1987a, Section 3], that is, it is a local height function $\lambda_{\mathbb{P}^{1} \times \mathbb{P}^{1}, \Delta}$, where $\Delta$ is the diagonal of $\mathbb{P}^{1} \times \mathbb{P}^{1}$.

The next lemma relates the logarithmic chordal metric $\lambda_{v}(x, y)$ to the usual metric $|x-y|_{v}$ arising from the absolute value $v$.

Lemma 3. Let $v \in M_{K}$ and let $\lambda_{v}$ be the logarithmic chordal metric on $\mathbb{P}^{1}\left(\mathbb{C}_{v}\right)$. Define $\ell_{v}=2$ if $v$ is archimedean, and $\ell_{v}=1$ if $v$ is nonarchimedean. Then for 
$x, y \in \mathbb{C}_{v}$ the inequality $\lambda_{v}(x, y)>\lambda_{v}(y, \infty)+\log \ell_{v}$ implies

$$
\lambda_{v}(y, \infty) \leq \lambda_{v}(x, y)+\log |x-y|_{v} \leq 2 \lambda_{v}(y, \infty)+\log \ell_{v} .
$$

Proof. Notice that by the definition of chordal metric,

$$
\lambda_{v}(x, y)=\lambda_{v}(x, \infty)+\lambda_{v}(y, \infty)-\log |x-y|_{v} .
$$

Therefore

$$
\lambda_{v}(x, y)+\log |x-y|_{v}=\lambda_{v}(x, \infty)+\lambda_{v}(y, \infty) \geq \lambda_{v}(y, \infty) .
$$

This gives the lower bound for the sum $\lambda_{v}(x, y)+\log |x-y|_{v}$.

For the upper bound, if $v$ is an archimedean place, then the assertion is the same as [Silverman 2007, Lemma 3.53]. We will not repeat the proof here. For the case where $v$ is nonarchimedean, notice that $\lambda_{v}$ satisfies the strong triangle inequality,

$$
\lambda_{v}(x, y) \geq \min \left(\lambda_{v}(x, z), \lambda_{v}(y, z)\right),
$$

and that this inequality is an equality if $\lambda_{v}(x, z) \neq \lambda_{v}(y, z)$. Suppose that $x$ and $y$ satisfy the condition required in the lemma, that is, $\lambda_{v}(x, y)>\lambda_{v}(y, \infty)$. (In this case, $\ell_{v}=1$.) We claim that $\lambda_{v}(x, \infty) \leq \lambda_{v}(y, \infty)$. Assume to the contrary that $\lambda_{v}(x, \infty)>\lambda_{v}(y, \infty)$. Then by the strong triangle inequality for $\lambda_{v}$, we have

$$
\lambda_{v}(x, y)=\min \left(\lambda_{v}(x, \infty), \lambda_{v}(y, \infty)\right)=\lambda_{v}(y, \infty) .
$$

But this contradicts the assumption that $\lambda_{v}(x, y)>\lambda_{v}(y, \infty)$. Now

$$
\begin{aligned}
\lambda_{v}(x, y)+\log |x-y|_{v} & =\lambda_{v}(x, \infty)+\lambda_{v}(y, \infty) \\
& \leq 2 \lambda_{v}(y, \infty) \text { by the claim, }
\end{aligned}
$$

which completes the proof of the lemma.

\section{Dynamics and height functions}

Let $\varphi: \mathbb{P}^{1} \rightarrow \mathbb{P}^{1}$ be a rational map on $\mathbb{P}^{1}$ of degree $d \geq 2$ defined over the number field $K$. We identify $K \cup\{\infty\} \simeq \mathbb{P}^{1}(K)$ by fixing an affine coordinate $z$ on $\mathbb{P}^{1}$, so $\alpha \in K$ equals $[\alpha, 1] \in \mathbb{P}^{1}(K)$, and the point at infinity is $[1,0]$. With respect to this affine coordinate, we identity rational maps $\varphi: \mathbb{P}^{1} \rightarrow \mathbb{P}^{1}$ with rational functions $\varphi(z) \in K(z)$.

Let $P \in \mathbb{P}^{1}$. Then the (forward) orbit of $P$ under iteration of $\varphi$ is the set

$$
\mathrm{O}_{\varphi}(P)=\left\{\varphi^{n}(P): n=0,1,2, \ldots\right\} .
$$

The point $P$ is called a wandering point of $\varphi$ if $\mathrm{O}_{\varphi}(P)$ is an infinite set; otherwise $P$ is called a preperiodic point of $\varphi$. The set of preperiodic points of $\varphi$ is denoted 
by $\operatorname{PrePer}(\varphi)$. We say that a point $A \in \mathbb{P}^{1}$ is an exceptional point if it is preperiodic and $\varphi^{-1}\left(\mathbb{O}_{\varphi}(A)\right)=\mathscr{O}_{\varphi}(A)$, which is equivalent to the assumption that the complete (forward and backward) $\varphi$-orbit of $A$ is a finite set. It is a standard fact that $A$ is an exceptional point for $\varphi$ if and only if $A$ a totally ramified fixed point of $\varphi^{2}$. (One direction is clear, and the other follows from the fact [Silverman 2007, Theorem 1.6] that if $A$ is an exceptional point, then $O_{\varphi}(A)$ consists of at most two points.)

For a point $P=\left[x_{0}, x_{1}\right] \in \mathbb{P}^{1}(K)$, the height of $P$ is

$$
h(P)=\sum_{v \in M_{K}} \frac{\left[K_{v}: \mathbb{Q}_{v}\right]}{[K: \mathbb{Q}]} \log \max \left(\left|x_{0}\right|_{v},\left|x_{1}\right|_{v}\right) .
$$

Then the canonical height of $P$ relative to the rational map $\varphi$ is given by the limit

$$
\hat{h}_{\varphi}(P)=\lim _{n \rightarrow \infty} h\left(\varphi^{n} P\right) / d^{n} .
$$

To simplify notation, we let $d_{v}=\left[K_{v}: \mathbb{Q}_{v}\right] /[K: \mathbb{Q}]$.

Using the definition of $\lambda_{v}$, we see that

$$
h(P)=\sum_{v \in M_{K}} d_{v} \lambda_{v}(P, \infty)+O(1) .
$$

More precisely, writing $P=\left[x_{0}, x_{1}\right]$ and $\infty=[1,0]$, we have

$$
h(P)=\sum_{v \in M_{K}^{0}} d_{v} \lambda_{v}(P, \infty)+\sum_{v \in M_{K}^{\infty}} d_{v} \log \left(\frac{\max \left\{\left|x_{0}\right|_{v},\left|x_{1}\right|_{v}\right\}}{\sqrt{\left|x_{0}\right|_{v}^{2}+\left|x_{1}\right|_{v}^{2}}}\right) .
$$

The quantity $\max \{a, b\} / \sqrt{a^{2}+b^{2}}$ is between $1 / \sqrt{2}$ and 1 for all nonnegative $a, b \in \mathbb{R}$, so

$$
-\frac{1}{2} \log 2 \leq h(P)-\sum_{v \in M_{K}} d_{v} \lambda_{v}(P, \infty) \leq 0 .
$$

For further material and basic properties of height functions, see [Silverman 2007, Sections 3.1-3.5].

For a polynomial $f=\sum a_{i} z^{i} \in K[z]$ and absolute value $v \in M_{K}$, we define

$$
|f|_{v}=\max \left\{\left|a_{i}\right|_{v}\right\} \quad \text { and } \quad h(f)=h\left(\left[\ldots, a_{i}, \ldots\right]\right)=\sum_{v \in M_{K}} d_{v} \log |f|_{v} .
$$

We say that a rational function $\varphi(z)=f(z) / g(z) \in K(z)$ of degree $d$ is written in normalized form if

$$
f(z)=\sum_{i=0}^{d} a_{i} z^{i} \quad \text { and } \quad g(z)=\sum_{i=0}^{d} b_{i} z^{i} \quad \text { with } a_{i}, b_{i} \in K,
$$


if $a_{d}$ and $b_{d}$ are not both zero, and if $f$ and $g$ are relatively prime in $K[z]$. For $v \in M_{K}$, we set $|\varphi|_{v}=\max \left\{|f|_{v},|g|_{v}\right\}$, and then the height of $\varphi$ is defined by

$$
h(\varphi)=h\left(\left[a_{0}, \ldots, a_{d}, b_{0}, \ldots, b_{d}\right]\right)=\sum_{v \in M_{K}} d_{v} \log |\varphi|_{v} .
$$

Directly from the definitions, we have

$$
\max (h(f), h(g)) \leq h(\varphi) .
$$

The following basic properties of absolute values of polynomials will be useful.

Lemma 4. Let $v \in M_{K}$ and let $f, g \in K[x]$ be polynomials with coefficients in $K$.

(a) $|f+g|_{v} \leq \begin{cases}|f|_{v}+|g|_{v} & \text { if } v \text { is archimedean, } \\ \max \left\{|f|_{v},|g|_{v}\right\} & \text { if } v \text { is nonarchimedean. }\end{cases}$

(b) Gauss's lemma. If $v$ is nonarchimedean, then $|f g|_{v}=|f|_{v}|g|_{v}$.

(c) If $v$ is archimedean and $\operatorname{deg} f+\operatorname{deg} g<d$, then

$$
4^{-d}|f g|_{v} \leq|f|_{v}|g|_{v} \leq 4^{d}|f g|_{v} .
$$

Proof. (a) follows from the definition. For (b) and (c), see for example [Lang 1983, Chapter 3, Propositions 2.1 and 2.3].

Proposition 5. Let $\left\{f_{1}, \ldots, f_{r}\right\}$ be a collection of polynomials in the ring $K[x]$.

(a) $h\left(f_{1} f_{2} \cdots f_{r}\right) \leq \sum_{i=1}^{r}\left(h\left(f_{i}\right)+\left(\operatorname{deg} f_{i}+1\right) \log 2\right)$

$$
\leq r \max _{1 \leq i \leq r}\left\{h\left(f_{i}\right)+\left(\operatorname{deg} f_{i}+1\right) \log 2\right\} .
$$

(b) $h\left(f_{1}+f_{2}+\cdots+f_{r}\right) \leq \sum_{i=1}^{r} h\left(f_{i}\right)+\log r$.

(c) Let $\varphi(z), \psi(z) \in K(z)$ be rational functions. Then

$$
h(\varphi \circ \psi) \leq h(\varphi)+(\operatorname{deg} \varphi) h(\psi)+(\operatorname{deg} \varphi)(\operatorname{deg} \psi) \log 8 .
$$

(d) Let $\varphi(z) \in K(z)$ be a rational function of degree $d \geq 2$. Then for all $n \geq 1$, we have

$$
h\left(\varphi^{n}\right) \leq\left(\frac{d^{n}-1}{d-1}\right) h(\varphi)+d^{2}\left(\frac{d^{n-1}-1}{d-1}\right) \log 8 .
$$

Proof. The proofs of (a) and (b) can be found in [Hindry and Silverman 2000, Proposition B.7.2], where the proposition is stated for multivariable polynomials. Since we'll use the arguments in (a) for the proof of (c), we repeat the proof of (a) for the one-variable case. (Also, our situation is slightly different from that in 
[Hindry and Silverman 2000], since we are using a projective height instead of an affine height.) Writing $f_{i}=\sum_{E} a_{i E} X^{E}$, we have

$$
f_{1} \cdots f_{r}=\sum_{E}\left(\sum_{e_{1}+\cdots+e_{r}=E} a_{1 e_{1}} \cdots a_{r e_{r}}\right) X^{E},
$$

and hence for $v \in M_{K}$,

$$
\left|f_{1} \cdots f_{r}\right|_{v}=\left.\left.\max _{E}\right|_{e_{1}+\cdots+e_{r}=E} a_{1 e_{1}} \cdots a_{r e_{r}}\right|_{v}
$$

and $h\left(f_{1} \cdots f_{r}\right)=\sum_{v \in M_{K}} d_{v} \log \left|f_{1} \cdots f_{r}\right|_{v}$. If $v$ is nonarchimedean, then by Gauss's lemma, Lemma 4(b), we have

$$
\left|f_{1} \cdots f_{r}\right|_{v}=\prod_{i=1}^{r}\left|f_{i}\right|_{v} .
$$

It remains to deal with an archimedean place $v$. We note that the number of terms in the sum appearing in the right side of (5) is $\left(\begin{array}{c}E+r-1 \\ E\end{array}\right)$. Hence

$$
\begin{aligned}
\left|f_{1} \cdots f_{r}\right|_{v} & \leq \max _{E}\left(\left(\begin{array}{c}
E+r-1 \\
E
\end{array}\right) \max _{e_{1}+\cdots+e_{r}=E}\left|a_{1 e_{1}} \cdots a_{r e_{r}}\right|_{v}\right) \\
& \leq \max _{E}\left(2^{E+r-1} \max _{e_{1}+\cdots+e_{r}=E}\left|a_{1 e_{1}} \cdots a_{r e_{r}}\right|_{v}\right) .
\end{aligned}
$$

Further, if $E>\operatorname{deg}\left(f_{1} \ldots f_{r}\right)$, then the product $a_{1 e_{1}} \cdots a_{r e_{r}}$ is zero, since in that case at least one of the $a_{i j}$ is zero. Hence

$$
\left|f_{1} \cdots f_{r}\right|_{v} \leq 2^{\operatorname{deg}\left(f_{1} \cdots f_{r}\right)+r-1} \prod_{i=1}^{r}\left|f_{i}\right|_{v} .
$$

Let $N_{v}=2^{\sum_{i}\left(\operatorname{deg} f_{i}+1\right)}$ if $v$ is archimedean, and $N_{v}=1$ if $v$ is nonarchimedean. Then we compute

$$
\begin{aligned}
h\left(f_{1} \cdots f_{r}\right) & =\sum_{v \in M_{K}} d_{v} \log \left|f_{1} \cdots f_{r}\right|_{v} \\
& \leq \sum_{v \in M_{K}} d_{v}\left(\log N_{v}+\log \prod_{i=1}^{r}\left|f_{i}\right|_{v}\right) \\
& \leq \sum_{i=1}^{r}\left(h\left(f_{i}\right)+\left(\operatorname{deg} f_{i}+1\right) \log 2\right) \\
& \leq r \max _{1 \leq i \leq r}\left\{h\left(f_{i}\right)+\left(\operatorname{deg} f_{i}+1\right) \log 2\right\},
\end{aligned}
$$

which completes the proof of (a). 
Next we give a proof of (c). Write $\psi=\psi_{0} / \psi_{1} \in K(z)$ in normalized form, so in particular $\psi_{0}$ and $\psi_{1}$ are relatively prime polynomials. Then

$$
(\varphi \circ \psi)(z)=\frac{\sum a_{i} \psi_{0}^{i} \psi_{1}^{d-i}}{\sum b_{i} \psi_{0}^{i} \psi_{1}^{d-i}},
$$

so by definition of the height of a rational function, we have

$$
h(\varphi \circ \psi) \leq \sum_{v \in M_{K}} d_{v} \log \max \left\{\left|\sum a_{i} \psi_{0}^{i} \psi_{1}^{d-i}\right|_{v},\left|\sum b_{i} \psi_{0}^{i} \psi_{1}^{d-i}\right|_{v}\right\} .
$$

For the right side of this inequality, if $v$ is nonarchimedean, then by Gauss's lemma again we have

$$
\left|\sum a_{i} \psi_{0}^{i} \psi_{1}^{d-i}\right|_{v} \leq \max \left(|f|_{v}\left|\psi_{0}\right|_{v}^{i}\left|\psi_{1}\right|_{v}^{d-i}\right) \leq|\varphi|_{v}|\psi|_{v}^{d} .
$$

Similarly,

$$
\left|\sum b_{i} \psi_{0}^{i} \psi^{d-i}\right|_{v} \leq|\varphi|_{v}|\psi|_{v}^{d} .
$$

Hence for $v$ nonarchimedean, $|\varphi \circ \psi|_{v} \leq|\varphi|_{v}|\psi|_{v}^{d}$.

Next let $v$ be an archimedean place of $K$. Then the triangle inequality gives

$$
\left|\sum a_{i} \psi_{0}^{i} \psi_{1}^{d-i}\right|_{v} \leq(d+1)|f|_{v} \max _{i}\left\{\left|\psi_{0}^{i} \psi_{1}^{d-i}\right|_{v}\right\} .
$$

Applying the estimate (6) to the product $\psi_{0}^{i} \psi_{1}^{d-i}$ yields

$$
\left|\psi_{0}^{i} \psi_{1}^{d-i}\right|_{v} \leq 2^{d(\operatorname{deg} \psi+1)}\left|\psi_{0}\right|_{v}^{i}\left|\psi_{1}\right|_{v}^{d-i} \leq 2^{d(\operatorname{deg} \psi+1)}|\psi|_{v}^{d} .
$$

Therefore,

$$
\left|\sum a_{i} \psi_{0}^{i} \psi_{1}^{d-1}\right|_{v} \leq(d+1) 2^{d(\operatorname{deg} \psi+1)}|f|_{v}|\psi|_{v}^{d} \leq(d+1) 2^{d(\operatorname{deg} \psi+1)}|\varphi|_{v}|\psi|_{v}^{d} .
$$

Similarly,

$$
\left|\sum b_{i} \psi_{0}^{i} \psi_{1}^{d-1}\right|_{v} \leq(d+1) 2^{d(\operatorname{deg} \psi+1)}|\varphi|_{v}|\psi|_{v}^{d} .
$$

We combine these estimates. To ease notation, we let $N_{v}=1$ for $v$ nonarchimedean and $N_{v}=(d+1) 2^{2 d \operatorname{deg} \psi}=(d+1) 4^{\operatorname{deg} \varphi \operatorname{deg} \psi}$ for $v$ archimedean. Then

$$
\begin{aligned}
h(\varphi \circ \psi) & \leq \sum_{v \in M_{K}} d_{v} \log \max \left\{\left|\sum a_{i} \psi_{0}^{i} \psi_{1}^{d-1}\right|_{v},\left|\sum b_{i} \psi_{0}^{i} \psi_{1}^{d-1}\right|_{v}\right\} \\
& \leq \sum_{v \in M_{K}} d_{v}\left(\log |\varphi|_{v}+d \log |\psi|_{v}+\log N_{v}\right) \\
& \leq h(\varphi)+d h(\psi)+(\operatorname{deg} \varphi)(\operatorname{deg} \psi) \log 4+\log (d+1) \\
& \leq h(\varphi)+d h(\psi)+(\operatorname{deg} \varphi)(\operatorname{deg} \psi) \log 8,
\end{aligned}
$$


since $d+1 \leq 2^{d} \leq 2^{d \operatorname{deg} \psi}$. This completes the proof of (c).

Finally, we prove (d) by induction on $n$. The stated inequality is clearly true for $n=1$. Assume now it is true for $n$. Then

$$
\begin{aligned}
h\left(\varphi^{n+1}\right) & \leq h\left(\varphi^{n}\right)+d^{n} h(\varphi)+d^{n+1} \log 8 \quad \text { from (c) applied to } \varphi^{n} \text { and } \varphi \\
& \leq\left(\frac{d^{n}-1}{d-1} h(\varphi)+d^{2} \frac{d^{n-1}-1}{d-1} \log 8\right)+d^{n} h(\varphi)+d^{n+1} \log 8 \\
\text { from the induction hypothesis } & \\
& =\left(\frac{d^{n+1}-1}{d-1}\right) h(\varphi)+d^{2}\left(\frac{d^{n}-1}{d-1}\right) \log 8 .
\end{aligned}
$$

The following facts about height functions are well known.

Proposition 6. Let $\varphi: \mathbb{P}^{1} \rightarrow \mathbb{P}^{1}$ be a rational map of degree $d \geq 2$ defined over $K$. There are constants $c_{1}, c_{2}, c_{3}$, and $c_{4}$, depending only on $d$, such that the following estimates hold for all $P \in \mathbb{P}^{1}(\bar{K})$.

(a) $|h(\varphi(P))-d h(P)| \leq c_{1} h(\varphi)+c_{2}$.

(b) $\left|\hat{h}_{\varphi}(P)-h(P)\right| \leq c_{3} h(\varphi)+c_{4}$.

(c) $\hat{h}_{\varphi}(\varphi(P))=d \hat{h}_{\varphi}(P)$.

(d) $P \in \operatorname{PrePer}(\varphi)$ if and only if $\hat{h}_{\varphi}(P)=0$.

Proof. See, for example, [Hindry and Silverman 2000, Sections B.2 and B.4] or [Silverman 2007, Section 3.4].

\section{A distance estimate}

Our goal in this section is a version of the inverse function theorem that gives explicit estimates for the dependence on the (local) heights of both the points and the function. It is undoubtedly possible to give a direct, albeit long and messy, proof of the desired result. We instead give a proof using universal families of maps and arithmetic distance functions. Before stating our result, we set notation for the universal family of degree $d$ rational maps on $\mathbb{P}^{1}$.

We write Rat $_{d} \subset \mathbb{P}^{2 d+1}$ for the space of rational maps of degree $d$, where we identify a rational map $\varphi=f / g=\sum a_{i} z^{i} / \sum b_{i} z^{i}$ with the point

$$
[\varphi]=[f, g]=\left[a_{0}, \ldots, a_{d}, b_{0}, \ldots, b_{d}\right] \in \mathbb{P}^{2 d+1} .
$$

If $\varphi \in \operatorname{Rat}_{d}(\overline{\mathbb{Q}})$ is defined over $\overline{\mathbb{Q}}$, we define the height of $\varphi$ as in Section 2 to be the height of the associated point in $\mathbb{P}^{2 d+1}(\overline{\mathbb{Q}})$ :

$$
h(\varphi)=h\left(\left[a_{0}, \ldots, a_{d}, b_{0}, \ldots, b_{d}\right]\right) .
$$

Over Rat $d$, there is a universal family of degree $d$ maps, which we denote by

$$
\Psi: \mathbb{P}^{1} \times \mathrm{Rat}_{d} \rightarrow \mathbb{P}^{1} \times \mathrm{Rat}_{d}, \quad(P, \psi) \mapsto(\psi(P), \psi) .
$$


We note that $\mathrm{Rat}_{d}$ is the complement in $\mathbb{P}^{2 d+1}$ of a hypersurface, which we denote by $\partial \operatorname{Rat}_{d}$. (The set $\partial \mathrm{Rat}_{d}$ is given by the resultant $\operatorname{Res}(f, g)=0$, so $\partial \operatorname{Rat}_{d}$ is a hypersurface of degree $2 d$.) Since $\mathbb{P}^{1}$ is complete, we have

$$
\partial\left(\mathbb{P}^{1} \times \operatorname{Rat}_{d}\right)=\mathbb{P}^{1} \times \partial \operatorname{Rat}_{d} .
$$

The map $\Psi$ is a finite map of degree $d$. Let $R(\Psi)$ denote its ramification locus. Looking at the behavior of $\Psi$ in a neighborhood of a point $(P, \psi)$, it is easy to see that the restriction of $R(\Psi)$ to a fiber $\mathbb{P}_{\psi}^{1}=\mathbb{P}^{1} \times\{\psi\}$ is the ramification divisor $\left.R(\Psi)\right|_{\mathbb{P}_{\psi}^{1}}=R(\psi)$ of $\psi$. So the ramification indices of the universal map $\Psi$ and a particular map $\psi$ are related by

$$
e_{(P, \psi)}(\Psi)=e_{P}(\psi) .
$$

Proposition 7. Let $\psi \in K(z)$ be a nontrivial rational function, let $S \subset M_{K}$ be a finite set of absolute values on $K$, each extended in some way to $\bar{K}$, and let $A, P \in \mathbb{P}^{1}(K)$. Then

$$
\sum_{v \in S} \max _{A^{\prime} \in \psi^{-1}(A)} e_{A^{\prime}}(\psi) d_{v} \lambda_{v}\left(P, A^{\prime}\right) \geq \sum_{v \in S} d_{v} \lambda_{v}(\psi(P), A)+O(h(A)+h(\psi)+1),
$$

where the implied constant depends only on the degree of the map $\psi$.

Proof. The statement and proof of Proposition 7 use the machinery of arithmetic distance functions and local height functions on quasiprojective varieties, as described in [Silverman 1987a], to which we refer for definitions, notation, and basic properties. We begin with the distribution relation for finite maps of smooth quasiprojective varieties [Silverman 1987a, Proposition 6.2(b)]. Applying this relation to the map $\Psi$ and points $x, y \in \mathbb{P}^{1} \times$ Rat $_{d}$ yields

$$
\delta(\Psi(x), y ; v)=\sum_{y^{\prime} \in \Psi^{-1}(y)} e_{y^{\prime}}(\Psi) \delta\left(x, y^{\prime} ; v\right)+O\left(\lambda_{\partial\left(\mathbb{P}^{1} \times \operatorname{Rat}_{d}\right)^{2}}(x, y ; v)\right),
$$

where $\delta(\cdot, \cdot ; v)$ is a $v$-adic arithmetic distance function on $\mathbb{P}^{1} \times \mathrm{Rat}_{d}$, and where $\lambda_{\partial\left(\mathbb{P}^{1} \times \mathrm{Rat}_{d}\right)^{2}}$ is a local height function for the indicated divisor. In particular, if we take $x=(P, \psi)$ and $y=(A, \psi)$, then the arithmetic distance function $\delta$ and the chordal metric $\lambda_{v}$ defined in Section 1 satisfy

$$
\begin{aligned}
\delta(\Psi(x), y ; v)=\delta(\Psi(P, \psi),(A, \psi) ; v) & =\delta((\psi(P), \psi),(A, \psi) ; v) \\
& =\lambda_{v}(\psi(P), A) .
\end{aligned}
$$

Similarly, if $y^{\prime}=\left(A^{\prime}, \psi\right) \in \Psi^{-1}(y)$, then

$$
\delta\left(x, y^{\prime} ; v\right)=\delta\left((P, \psi),\left(A^{\prime}, \psi\right) ; v\right)=\lambda_{v}\left(P, A^{\prime}\right) .
$$


Further, since $\partial\left(\mathbb{P}^{1} \times \mathrm{Rat}_{d}\right)=\mathbb{P}^{1} \times \partial \mathrm{Rat}_{d}$ is the pull-back of a divisor on $\mathrm{Rat}_{d}$ and $\partial\left(\mathbb{P}^{1} \times \mathrm{Rat}_{d}\right)^{2}=\left(\mathbb{P}^{1} \times \partial \mathrm{Rat}_{d}\right) \times\left(\mathbb{P}^{1} \times \mathrm{Rat}_{d}\right)+\left(\mathbb{P}^{1} \times \mathrm{Rat}_{d}\right) \times\left(\mathbb{P}^{1} \times \partial \mathrm{Rat}_{d}\right)$, applying [Silverman 1987a, Proposition 5.3(a)] gives

$$
\begin{aligned}
\lambda_{\partial\left(\mathbb{P}^{1} \times \operatorname{Rat}_{d}\right)^{2}}(x, y ; v) & \gg \ll \lambda_{\mathbb{P}^{1} \times \partial \operatorname{Rat}_{d}}((P, \psi) ; v)+\lambda_{\mathbb{P}^{1} \times \partial \operatorname{Rat}_{d}}((A, \psi) ; v) \\
\gg & \ll \lambda_{\partial \operatorname{Rat}_{d}}(\psi ; v) .
\end{aligned}
$$

Substituting (7), (9), and (10) into the distribution relation (8) yields

$$
\lambda_{v}(\psi(P), A)=\sum_{A^{\prime} \in \psi^{-1}(A)} e_{A^{\prime}}(\psi) \lambda_{v}\left(P, A^{\prime}\right)+O\left(\lambda_{\partial \operatorname{Rat}_{d}}(\psi ; v)\right) .
$$

To ease notation, let $A_{v}^{\prime} \in \psi^{-1}(A)$ be a point satisfying

$$
e_{A_{v}^{\prime}}(\psi) \lambda_{v}\left(P, A_{v}^{\prime}\right)=\max _{A^{\prime} \in \psi^{-1}(A)} e_{A^{\prime}} \lambda_{v}\left(P, A^{\prime}\right)
$$

Then for any $A^{\prime} \in \psi^{-1}(A)$ we have

$$
\begin{array}{rlrl}
e_{A^{\prime}}(\psi) \lambda_{v}\left(P, A^{\prime}\right) & =\min \left\{e_{A_{v}^{\prime}}(\psi) \lambda_{v}\left(P, A_{v}^{\prime}\right), e_{A^{\prime}}(\psi) \lambda_{v}\left(P, A^{\prime}\right)\right\} \\
& & \text { from the choice of } A_{v}^{\prime} \\
& \leq d \min \left\{\lambda_{v}\left(P, A_{v}^{\prime}\right), \lambda_{v}\left(P, A^{\prime}\right)\right\} & \text { since } \psi \text { has degree } d \\
& \leq d \lambda_{v}\left(A_{v}^{\prime}, A^{\prime}\right)+O(1) & & \text { from the triangle inequality. }
\end{array}
$$

This is a nontrivial estimate for $A^{\prime} \neq A_{v}^{\prime}$, so in (11) we pull off the $A_{v}^{\prime}$ term and use (12) for the other terms to obtain

$$
\lambda_{v}(\psi(P), A) \leq e_{A_{v}^{\prime}}(\psi) \lambda_{v}\left(P, A_{v}^{\prime}\right)+d \sum_{\substack{A^{\prime} \in \psi^{-1}(A) \\ A^{\prime} \neq A_{v}^{\prime}}} \lambda_{v}\left(A_{v}^{\prime}, A^{\prime}\right)+O\left(\lambda_{\partial \operatorname{Rat}_{d}}(\psi ; v)\right)
$$

The next lemma gives an upper bound for $\lambda_{v}\left(A_{v}^{\prime}, A^{\prime}\right)$.

Lemma 8. There is a constant $C=C(d)$ such that the following holds. Let $\psi \in \operatorname{Rat}_{d}(\overline{\mathbb{Q}})$, let $A \in \mathbb{P}^{1}(\overline{\mathbb{Q}})$, and let $A^{\prime}, A^{\prime \prime} \in \psi^{-1}(A)$ be distinct points. Then

$$
\sum_{v \in M_{K}} d_{v} \lambda_{v}\left(A^{\prime}, A^{\prime \prime}\right) \leq C(h(A)+h(\psi)+1) .
$$

Proof. In the notation of [Silverman 1987a], we have

$$
\begin{aligned}
\lambda_{v}\left(A^{\prime}, A^{\prime \prime}\right) & =\delta_{\mathbb{P}^{1} \times \operatorname{Rat}_{d}}\left(\left(A^{\prime}, \psi\right),\left(A^{\prime \prime}, \psi\right) ; v\right) \\
& =\lambda_{\left(\mathbb{P}^{1} \times \operatorname{Rat}_{d}\right)^{2}, \Delta}\left(\left(A^{\prime}, \psi\right),\left(A^{\prime \prime}, \psi\right) ; v\right),
\end{aligned}
$$


where $\Delta$ is the diagonal of $\left(\mathbb{P}^{1} \times \operatorname{Rat}_{d}\right)^{2}$. Summing over $v$ gives height functions

$$
\begin{aligned}
\sum_{v \in M_{K}} \lambda_{v}\left(A^{\prime}, A^{\prime \prime}\right)=h_{\left(\mathbb{P}^{1} \times \mathrm{Rat}_{d}\right)^{2}, \Delta}\left(\left(A^{\prime}, \psi\right)\right. & \left.,\left(A^{\prime \prime}, \psi\right)\right) \\
& +O\left(h_{\partial\left(\mathbb{P}^{1} \times \operatorname{Rat}_{d}\right)^{2}}\left(\left(A^{\prime}, \psi\right),\left(A^{\prime \prime}, \psi\right)\right)\right)+1 .
\end{aligned}
$$

Choosing an ample divisor $H$ on $\mathbb{P}^{1} \times$ Rat $_{d}$, we use the fact that heights with respect to a subscheme are dominated by ample heights away from the support of the subscheme [Silverman 1987a, Proposition 4.2]. (This is where we use the assumption that $A^{\prime} \neq A^{\prime \prime}$, which ensures that the point $\left(\left(A^{\prime}, \psi\right),\left(A^{\prime \prime}, \psi\right)\right)$ is not on the diagonal.) This yields

$$
\begin{aligned}
\sum_{v \in M_{K}} \lambda_{v}\left(A^{\prime}, A^{\prime \prime}\right) & \ll h_{\mathbb{P}^{1} \times \operatorname{Rat}_{d}, H}\left(A^{\prime}, \psi\right)+h_{\mathbb{P}^{1} \times \operatorname{Rat}_{d}, H}\left(A^{\prime \prime}, \psi\right)+1 \\
& \ll h\left(A^{\prime}\right)+h\left(A^{\prime \prime}\right)+h(\psi)+1 .
\end{aligned}
$$

We now use [Silverman 2009, Theorem 2], which says that there are positive constants $C_{1}, C_{2}, C_{3}$, depending only on the degree of $\psi$, such that

$$
h(\psi(P)) \geq C_{1} h(P)-C_{2} h(\psi)-C_{3} .
$$

(The paper [Silverman 2009] deals with general rational maps $\mathbb{P}^{n} \rightarrow \mathbb{P}^{n}$. In our case with $n=1$, it would be a tedious, but not difficult, calculation to give explicit values for the $C_{i}$, including of course $C_{1}=\operatorname{deg} \psi$.) Applying (15) with $P=A^{\prime}$ and $P=A^{\prime \prime}$, we substitute into (14) to obtain

$$
\sum_{v \in M_{K}} \lambda_{v}\left(A^{\prime}, A^{\prime \prime}\right) \ll h(A)+h(\psi)+1,
$$

which completes the proof of Lemma 8 .

We use Lemma 8 to bound the sum in the right side of the inequality (13). We note that $\lambda_{v}\left(A^{\prime}, A^{\prime \prime}\right) \geq 0$ for all points, so the lemma implies in particular that $\sum_{v \in S} d_{v} \lambda_{v}\left(A^{\prime}, A^{\prime \prime}\right) \ll h(A)+h(\psi)+1$ for any set of places $S$. Further, the sum in (13) has at most $d-1$ terms. Hence we obtain

$$
\sum_{v \in S} d_{v} \lambda_{v}(\psi(P), A) \leq \sum_{v \in S} e_{A_{v}^{\prime}}(\psi) d_{v} \lambda_{v}\left(P, A_{v}^{\prime}\right)+O(h(A)+h(\psi)+1) .
$$

In this inequality, the $O(h(\psi))$ term comes from two places, Lemma 8 and

$$
\sum_{v \in S} d_{v} \lambda_{\partial \operatorname{Rat}_{d}}(\psi ; v) \leq \sum_{v \in M_{K}} d_{v} \lambda_{\partial \operatorname{Rat}_{d}}(\psi ; v)=h_{\partial \operatorname{Rat}_{d}}(\psi)=O(h(\psi)+1),
$$

where the last equality comes from the fact that $\partial \mathrm{Rat}_{d}$ is a hypersurface of degree $2 d$ in $\mathbb{P}^{2 d+1}$. This completes the proof of Proposition 7 . 


\section{A ramification estimate and a quantitative version of Roth's theorem}

In this section we state two known results that will be needed to prove our main theorem. The first says that away from exceptional points, the ramification of $\varphi^{m}$ tends to spread out as $m$ increases.

Lemma 9. Fix an integer $d \geq 2$. There exist constants $\kappa_{1}$ and $\kappa_{2}<1$, depending only on $d$, such that for all degree $d$ rational maps $\varphi: \mathbb{P}^{1} \rightarrow \mathbb{P}^{1}$, all points $Q \in \mathbb{P}^{1}$ that are not exceptional for $\varphi$, all integers $m \geq 1$, and all $P \in \varphi^{-m}(Q)$, we have

$$
e_{P}\left(\varphi^{m}\right) \leq \kappa_{1}\left(\kappa_{2} d\right)^{m} \text {. }
$$

Proof. This is [Silverman 2007, Lemma 3.52]; see in particular the last paragraph of the proof. It is not difficult to give explicit values for the constants. If $Q$ is not preperiodic, then the stronger estimate $e_{P}\left(\varphi^{m}\right) \leq e^{2 d-2}$ is true for all $m$.

The second result is the following quantitative version of Roth's theorem.

Theorem 10. Let $S$ be a finite subset of $M_{K}$ that contains all infinite places. We assume that each place in $S$ is extended to $\bar{K}$ in some fashion. Set the following notation.

$\begin{array}{ll}s & \text { the cardinality of } S . \\ \Upsilon & \text { a finite, } G_{\bar{K} / K} \text {-invariant subset of } \bar{K} . \\ \beta & \text { a map } S \rightarrow \Upsilon . \\ \mu>2 & \text { a constant. } \\ M \geq 0 & \text { a constant. }\end{array}$

There are constants $r_{1}$ and $r_{2}$, depending only on $[K: \mathbb{Q}], \# \Upsilon$, and $\mu$, such that there are at most $4^{s} r_{1}$ elements $x \in K$ satisfying both of the following conditions:

$$
\begin{gathered}
\sum_{v \in S} d_{v} \log ^{+}\left|x-\beta_{v}\right|_{v}^{-1} \geq \mu h(x)-M . \\
h(x) \geq r_{2} \max _{v \in S}\left\{h\left(\beta_{v}\right), M, 1\right\} .
\end{gathered}
$$

Proof. This is [Silverman 1987b, Theorem 2.1], with a small change of notation. For explicit values of the constants, see [Gross 1990].

\section{A bound for the number of quasiintegral points in an orbit}

In this section we prove our main result, which is an explicit upper bound for the number of iterates $\varphi^{n}(P)$ that are close to a given base point $A$ in any one of a fixed finite number of $v$-adic topologies. Here is the precise statement.

Theorem 11. Let $\varphi \in K(z)$ be a rational map of degree $d \geq 2$. Fix a point $A \in \mathbb{P}^{1}(K)$ that is not an exceptional point for $\varphi$, and let $P \in \mathbb{P}^{1}(K)$ be a wandering 
point for $\varphi$. For any finite set of places $S \subset M_{K}$ and any constant $1 \geq \varepsilon>0$, define a set of nonnegative integers by

$$
\Gamma_{\varphi, S}(A, P, \varepsilon)=\left\{n \geq 0: \sum_{v \in S} d_{v} \lambda_{v}\left(\varphi^{n} P, A\right) \geq \varepsilon \hat{h}_{\varphi}\left(\varphi^{n} P\right)\right\} .
$$

(a) There exist constants

$$
\gamma_{1}=\gamma_{1}(d, \varepsilon,[K: \mathbb{Q}]) \quad \text { and } \quad \gamma_{2}=\gamma_{2}(d, \varepsilon,[K: \mathbb{Q}])
$$

such that

$$
\#\left\{n \in \Gamma_{\varphi, S}(A, P, \varepsilon): n>\gamma_{1}+\log _{d}^{+}\left(\frac{h(\varphi)+\hat{h}_{\varphi}(A)}{\hat{h}_{\varphi}(P)}\right)\right\} \leq 4^{\# S} \gamma_{2} .
$$

(b) In particular, there is a constant $\gamma_{3}=\gamma_{3}(d, \varepsilon,[K: \mathbb{Q}])$ such that

$$
\# \Gamma_{\varphi, S}(A, P, \varepsilon) \leq 4^{\# S} \gamma_{3}+\log _{d}^{+}\left(\frac{h(\varphi)+\hat{h}_{\varphi}(A)}{\hat{h}_{\varphi}(P)}\right) .
$$

(c) There is a constant $\gamma_{4}=\gamma_{4}(K, S, \varphi, A, \epsilon)$ that is independent of $P$ such that

$$
\max \Gamma_{\varphi, S}(A, P, \varepsilon) \leq \gamma_{4} .
$$

Before giving the proof of Theorem 11, we make a number of remarks.

Remark 12. Note that as a consequence of Proposition 6(d), we have $\hat{h}_{\varphi}(P)>0$ if $P$ is wandering point for $\varphi$. Hence the right side of (19) is well-defined.

Remark 13. If we take $\varepsilon=1$, then the set $\Gamma_{\varphi, S}(A, P, \varepsilon)$ more or less coincides with the set of points in the orbit $\mathrm{O}_{\varphi}(P)$ that are $S$-integral with respect to $A$. We say "more or less" because $\Gamma_{\varphi, S}(A, P, \varepsilon)$ is defined using the canonical height of $\varphi^{n}(P)$, rather than the naive height. But using the inequality $\left|\hat{h}_{\varphi}(P)-h(P)\right| \ll$ $h(\varphi)+1$ from Proposition 6 and adjusting the constants, it is not hard to see that the estimate (19) remains true for the set

$$
\Gamma_{\varphi, S}^{\text {naive }}(A, P, \varepsilon)=\left\{n \geq 0: \sum_{v \in S} d_{v} \lambda_{v}\left(\varphi^{n} P, A\right) \geq \varepsilon h\left(\varphi^{n} P\right)\right\} .
$$

(See the proof of Corollary 17.) For example, taking $A=\infty$, the set $\Gamma_{\varphi, S}^{\text {naive }}(A, P, \varepsilon)$ consists of the points $\varphi^{n}(P)$ such that $z\left(\varphi^{n}(P)\right)$ is $\left(S, \varepsilon_{0}\right)$-integral for some $\varepsilon_{0}$. This is the motivation for saying that the points in $\Gamma_{\varphi, S}(A, P, \varepsilon)$ are quasi- $(S, \varepsilon)$-integral with respect to $A$, where $\varepsilon$ measures the degree of $S$-integrality.

Remark 14. The dependence of the bounds (18) and (19) on $h(\varphi), \hat{h}_{\varphi}(A)$, and $\hat{h}_{v}(P)$ are quite interesting. A dynamical analogue of a conjecture of Lang asserts that the ratio $h(\varphi) / \hat{h}_{\varphi}(P)$ is bounded, independently of $\varphi$ and $P$, provided that $\varphi$ is suitably minimal with respect to $\mathrm{PGL}_{2}(K)$-conjugation. See [Silverman 2007, Conjecture 4.98]. 
On the other hand, there cannot be a uniform bound for the ratio $\hat{h}_{\varphi}(A) / \hat{h}_{\varphi}(P)$, since $A$ and $P$ may be chosen arbitrarily and independent of one another. This raises the interesting question of whether the bound for $\# \Gamma_{\varphi, S}(A, P, \varepsilon)$ actually needs to depend on $A$. Even in very simple situations, it appears difficult to answer this question. For example, consider the map $\varphi(z)=z^{2}$, the initial point $P=2$, and the set of primes $S=\{\infty, 3,5\}$. As $A \in \mathbb{Q}^{*}$ varies, is it possible for the orbit $\mathrm{O}_{\varphi}(P)$ to contain more and more points that are $S$-integral with respect to $A$ ? Writing $A=x / y$, we are asking if

$$
\sup _{x, y \in \mathbb{Z}} \#\left\{(n, i, j) \in \mathbb{N}^{3}: y \cdot 2^{2^{n}}-x=3^{i} 5^{j}\right\}=\infty .
$$

Remark 15. We observe that $\# \Gamma_{\varphi, S}(A, P, \varepsilon)$ can grow as fast as $\log \left(\varepsilon^{-1}\right)$ as $\varepsilon \rightarrow 0^{+}$. For example, consider the map $\varphi(z)=z^{d}+z^{d-1}$, the points $A=0$ and $P=p$, and the set of primes $S=\{p\}$. Since $\varphi^{n}(z)=z^{(d-1)^{n}}+$ higher order terms, we have $\left|\varphi^{n}(p)\right|_{p}=p^{-(d-1)^{n}}$, so

$$
\lambda_{p}\left(\varphi^{n} P, A\right)=\lambda_{p}\left(\varphi^{n}(p), 0\right)=-\log \left|\varphi^{n}(p)\right|_{p}=(d-1)^{n} \log p .
$$

Thus $\Gamma_{\varphi, S}(A, P, \varepsilon)$ consists of all $n \geq 0$ satisfying

$$
(d-1)^{n} \log p \geq \varepsilon \hat{h}_{\varphi}\left(\varphi^{n} P\right)=\varepsilon d^{n} \hat{h}_{\varphi}(P) .
$$

Hence

$$
\begin{aligned}
\# \Gamma_{\varphi, S}(A, P, \varepsilon) & =\left\lfloor\log \left(\frac{\log p}{\varepsilon \hat{h}_{\varphi}(P)}\right) / \log \left(\frac{d}{d-1}\right)\right\rfloor+1 \\
& =\frac{\log \left(\varepsilon^{-1}\right)}{\log (d /(d-1))}+o\left(\log \varepsilon^{-1}\right) \quad \text { as } \epsilon \rightarrow 0^{+} .
\end{aligned}
$$

In particular, if $\varepsilon$ is small and $d$ is large, so $\log (d /(d-1)) \approx 1 /(d-1)$, then we have

$$
\# \Gamma_{\varphi, S}(A, P, \varepsilon) \approx(d-1) \log \left(\epsilon^{-1}\right) .
$$

Remark 16. See [Gross and Silverman 1995; Silverman 1987b] for a version of Theorem 11 for elliptic curves. These papers deal with points on an elliptic curve $E$ that are quasi- $(S, \epsilon)$-integral with respect to $O$, the zero point of $E$. It is also of interest to study points that are integral with respect to some other point $A$, and in particular to see how the bound depends on $A$. The distance function on $E$ is translation invariant up to $O(h(E))$, so we want to estimate the size of the set

$$
\left\{P \in E(K): \sum_{v \in S} d_{v} \lambda_{v}(P-A) \geq \varepsilon \hat{h}_{E}(P)\right\} .
$$

Translating the points in (20) by $A$, we want to count points satisfying $\sum d_{v} \lambda_{v}(P) \geq$ $\varepsilon \hat{h}_{E}(P+A)+O(h(E))$. The canonical height on an elliptic curve is a quadratic form, so $\hat{h}_{E}(P+A) \leq 2 \hat{h}_{E}(P)+2 \hat{h}_{E}(A)$. Using the results in [Silverman 1987b], 
this leads to a bound for the set (20) in which the dependence on $A$ appears as the ratio $\hat{h}_{E}(A) / \hat{h}_{E}\left(P_{\min }\right)$, where $P_{\min }$ is the point of smallest nonzero height in $E(K)$. This is analogous to the dependence on $A$ in (19).

Proof of Theorem 11. For brevity, we will write $\Gamma_{S}(\varepsilon)$ in place of $\Gamma_{\varphi, S}(A, P, \varepsilon)$. For the given $\varepsilon$, we set $m \geq 1$ to be the smallest integer satisfying $\kappa_{2}^{m} \leq \varepsilon / 5 \kappa_{1}$, where $\kappa_{1}$ and $\kappa_{2}$ are the positive constants appearing in Lemma 9. Since $\kappa_{2}<1$, there exists such an integer $m$. Notice that $\kappa_{1}$ and $\kappa_{2}$ depend only on $d$; consequently $m$ depends only on $d$ and $\varepsilon$. More precisely, if we assume (without loss of generality) that $\varepsilon<1 / 2$, then $m \ll \log \left(\varepsilon^{-1}\right)$, where the implied constant depends only on $d$.

Put

$$
\mathbf{e}_{m}=\max _{A^{\prime} \in \varphi^{-m}(A)} e_{A^{\prime}}\left(\varphi^{m}\right) .
$$

Then Lemma 9 and our choice of $m$ imply that

$$
\mathbf{e}_{m} \leq \kappa_{1}\left(\kappa_{2} d\right)^{m} \leq \varepsilon d^{m} / 5 .
$$

Further, Proposition 7 says that for all $Q \in \mathbb{P}^{1}(K)$ we have

$$
\mathbf{e}_{m} \sum_{v \in S} \max _{A^{\prime} \in \varphi^{-m}(A)} d_{v} \lambda_{v}\left(Q, A^{\prime}\right) \geq \sum_{v \in S} d_{v} \lambda_{v}\left(\varphi^{m} Q, A\right)-O\left(h(A)+h\left(\varphi^{m}\right)+1\right),
$$

where the implied constant depends on $\operatorname{deg}\left(\varphi^{m}\right)$.

Suppose first that $n \leq m$ for all $n \in \Gamma_{S}(\varepsilon)$. Then clearly $\# \Gamma_{S}(\varepsilon) \leq m$, and from our choice of $m$ we have

$$
\# \Gamma_{S}(\varepsilon) \leq m \leq \frac{\log \left(5 \kappa_{1}\right)+\log \left(\varepsilon^{-1}\right)}{\log \left(\kappa_{2}^{-1}\right)}+1 .
$$

This upper bound has the desired form, since $\kappa_{1}>0$ and $1>\kappa_{2}>0$ depend only on $d$.

We may thus assume that there exists an $n \in \Gamma_{S}(\varepsilon)$ such that $n>m$, and we fix such an $n$. By the definition of $\Gamma_{S}(\varepsilon)$ we have

$$
\varepsilon \hat{h}_{\varphi}\left(\varphi^{n} P\right) \leq \sum_{v \in S} d_{v} \lambda_{v}\left(\varphi^{n} P, A\right) .
$$

Applying (22) to the point $Q=\varphi^{n-m}(P)$ yields

$$
\varepsilon \hat{h}_{\varphi}\left(\varphi^{n} P\right) \leq \mathbf{e}_{m} \sum_{v \in S} d_{v} \max _{A^{\prime} \in \varphi^{-m}(A)} \lambda_{v}\left(\varphi^{n-m} P, A^{\prime}\right)+O\left(h(A)+h\left(\varphi^{m}\right)+1\right),
$$

where the big- $O$ constant depends on $\operatorname{deg} \varphi^{m}=d^{m}$, and so on $d$ and $\varepsilon$.

For each $v \in S$ we choose an $A_{v}^{\prime} \in \varphi^{-m}(A)$ satisfying

$$
\lambda_{v}\left(\varphi^{n-m} P, A_{v}^{\prime}\right)=\max _{A^{\prime} \in \varphi^{-m} A} \lambda_{v}\left(\varphi^{n-m} P, A^{\prime}\right) .
$$


(For ease of exposition, we will assume that $z\left(A^{\prime}\right) \neq \infty$ for all $A^{\prime} \in \varphi^{-m} A$. If this is not the case, then we use $z$ for some of the $A^{\prime}$, and we use $z^{-1}$ for the others.)

Let $S^{\prime} \subset S$ be the set of places in $S$ defined by

$$
S^{\prime}=\left\{v \in S: \lambda_{v}\left(\varphi^{n-m}(P), A_{v}^{\prime}\right)>\lambda_{v}\left(A_{v}^{\prime}, \infty\right)+\log \ell_{v}\right\}
$$

where we recall that $\ell_{v}=2$ if $v$ is archimedean and $\ell_{v}=1$ otherwise. Set $S^{\prime \prime}=S \backslash S^{\prime}$. Applying Lemma 3 to the places in $S^{\prime}$ and using the definition of $S^{\prime \prime}$ for the places in $S^{\prime \prime}$, we find that

$$
\begin{aligned}
& \varepsilon \hat{h}_{\varphi}\left(\varphi^{n}(P)\right) \\
& \leq\left(\sum_{v \in S^{\prime}}+\sum_{v \in S^{\prime \prime}}\right) d_{v} \lambda_{v}\left(\varphi^{n} P, A\right) \\
& \leq \mathbf{e}_{m}\left(\sum_{v \in S^{\prime}}+\sum_{v \in S^{\prime \prime}}\right) d_{v} \lambda_{v}\left(\varphi^{n-m}(P), A_{v}^{\prime}\right)+O\left(h(A)+h\left(\varphi^{m}\right)+1\right) \\
& \quad \text { from the definition of } A_{v}^{\prime} \text { and (23) } \\
& \leq \mathbf{e}_{m} \sum_{v \in S^{\prime}} d_{v}\left(2 \lambda_{v}\left(A_{v}^{\prime}, \infty\right)-\log \left|z\left(\varphi^{n-m}(P)\right)-z\left(A_{v}^{\prime}\right)\right|+\log \ell_{v}\right) \\
& \quad+\mathbf{e}_{m} \sum_{v \in S^{\prime \prime}} d_{v}\left(\lambda_{v}\left(A_{v}^{\prime}, \infty\right)+\log \ell_{v}\right)+O\left(h(A)+h\left(\varphi^{m}\right)+1\right) \\
& \quad \text { from Lemma } 3 \\
& \quad \leq \mathbf{e}_{m} \sum_{v \in S^{\prime}} d_{v} \log \left|z\left(\varphi^{n-m}(P)\right)-z\left(A_{v}^{\prime}\right)\right|^{-1} \\
& \quad+\mathbf{e}_{m} \sum_{v \in S} d_{v}\left(2 \lambda_{v}\left(A_{v}^{\prime}, \infty\right)+\log \ell_{v}\right)+O\left(h(A)+h\left(\varphi^{m}\right)+1\right) .
\end{aligned}
$$

We now use (b) and (c) of Proposition 6 to observe that

$$
\begin{aligned}
\sum_{v \in S} d_{v} \lambda_{v}\left(A_{v}^{\prime}, \infty\right) & \leq \sum_{A^{\prime} \in \varphi^{-m}(A)} \sum_{v \in S} d_{v} \lambda_{v}\left(A^{\prime}, \infty\right) \leq \sum_{A^{\prime} \in \varphi^{-m}(A)} h\left(A^{\prime}\right) \\
& \leq \sum_{A^{\prime} \in \varphi^{-m}(A)}\left(\hat{h}_{\varphi}\left(A^{\prime}\right)+O(h(\varphi)+1)\right) \\
& \leq \hat{h}_{\varphi}(A)+O(h(\varphi)+1),
\end{aligned}
$$

Here the last line follows because there are at most $d^{m}$ terms in the sum, and $\hat{h}_{\varphi}\left(A^{\prime}\right)=d^{-m} \hat{h}_{\varphi}(A)$. The constants depend only on $m$ and $d$, and so on $\varepsilon$ and $d$. Further, from the definition of $\ell_{v}$, we have

$$
\sum_{v \in S} d_{v} \log \ell_{v} \leq \log 2
$$


We also note from Proposition 5(d) that $h\left(\varphi^{m}\right) \ll h(\varphi)+1$, with the implied constant depending only on $d$ and $m$. Hence

$$
\begin{aligned}
\varepsilon \hat{h}_{\varphi}\left(\varphi^{n}(P)\right) \leq \mathbf{e}_{m} \sum_{v \in S^{\prime}} d_{v} \log ^{+}\left|z\left(\varphi^{n-m}(P)\right)-z\left(A_{v}^{\prime}\right)\right|^{-1} & \\
& +O\left(\hat{h}_{\varphi}(A)+h(\varphi)+1\right) .
\end{aligned}
$$

We are going to apply Roth's theorem (Theorem 10) to the set

$$
\Upsilon=\left\{z\left(A^{\prime}\right): A^{\prime} \in \varphi^{-m}(A)\right\} \subset \bar{K},
$$

the map $\beta: S^{\prime} \rightarrow \Upsilon$ given by $\beta_{v}=A_{v}^{\prime}$, and the points $x=\varphi^{n-m}(P)$ for $n \in \Gamma_{S}(\epsilon)$. We note that $\Upsilon$ is a $G_{\bar{K} / K}$-invariant set and that \# $\leq d^{m}$. We apply Theorem 10 to the set of places $S^{\prime}$, taking $M=0$ and $\mu=5 / 2$. This gives constants $r_{1}$ and $r_{2}$, depending only on $[K: \mathbb{Q}], d$, and $\varepsilon$, such that the set of $n \in \Gamma_{S}(\epsilon)$ with $n>m$ can be written as a union

$$
\left\{n \in \Gamma_{S}(\epsilon): n>m\right\}=T_{1} \cup T_{2} \cup T_{3},
$$

whose three sets are characterized as follows:

$$
\begin{aligned}
\# T_{1} & \leq 4^{\# S^{\prime}} r_{1}, \\
T_{2} & =\left\{n>m: \sum_{v \in S^{\prime}} d_{v} \log ^{+}\left|z\left(\varphi^{n-m}(P)\right)-z\left(A_{v}^{\prime}\right)\right|^{-1} \leq \frac{5}{2} h\left(\varphi^{n-m}(P)\right)\right\}, \\
T_{3} & =\left\{n>m: h\left(\varphi^{n-m}(P)\right) \leq r_{2} \max _{v \in S^{\prime}}\left\{h\left(A_{v}^{\prime}\right), 1\right\}\right\} .
\end{aligned}
$$

We already have a bound for the size of $T_{1}$, so we look at $T_{2}$ and $T_{3}$. We start with $T_{3}$ and use (b) and (c) of Proposition 6 to estimate

$$
\begin{aligned}
h\left(A_{v}^{\prime}\right) & \leq \hat{h}_{\varphi}\left(A^{\prime}\right)+c_{3} h(\varphi)+c_{4} \\
& =d^{-m} \hat{h}_{\varphi}(A)+c_{3} h(\varphi)+c_{4}, \\
h\left(\varphi^{n-m}(P)\right) & \geq \hat{h}_{\varphi}\left(\varphi^{n-m}(P)\right)-c_{3} h(\varphi)-c_{4} \\
& =d^{n-m} \hat{h}_{\varphi}(P)-c_{3} h(\varphi)-c_{4} .
\end{aligned}
$$

Hence

$$
T_{3} \subset\left\{n>m: d^{n-m} \hat{h}_{\varphi}(P) \leq c_{5} \hat{h}_{\varphi}(A)+c_{6} h(\varphi)+c_{7}\right\},
$$

so every $n \in T_{3}$ satisfies

$$
\begin{aligned}
n & \leq m+\log _{d}^{+}\left(\frac{c_{5} \hat{h}_{\varphi}(A)+c_{6} h(\varphi)+c_{7}}{\hat{h}_{\varphi}(P)}\right) \\
& \leq c_{8}+\log _{d}^{+}\left(\frac{\hat{h}_{\varphi}(A)+h(\varphi)}{\hat{h}_{\varphi}(P)}\right) .
\end{aligned}
$$


Finally, we consider the set $T_{2}$. Again using (b) and (c) of Proposition 6 to relate $h\left(\varphi^{n-m}(P)\right)$ to $d^{n-m} \hat{h}_{\varphi}(P)$, we find that every $n \in T_{2}$ satisfies

$$
\sum_{v \in S^{\prime}} d_{v} \log ^{+}\left|z\left(\varphi^{n-m}(P)\right)-z\left(A_{v}^{\prime}\right)\right|^{-1} \leq \frac{5}{2} d^{n-m} \hat{h}_{\varphi}(P)+c_{3} h(\varphi)+c_{4} .
$$

We substitute this estimate into (24) to obtain

$$
\varepsilon \hat{h}_{\varphi}\left(\varphi^{n}(P)\right) \leq \mathbf{e}_{m} \frac{5}{2} d^{n-m} \hat{h}_{\varphi}(P)+c_{9}\left(\hat{h}_{\varphi}(A)+h(\varphi)+1\right) .
$$

We know from (21) that $\mathbf{e}_{m} \leq \varepsilon d^{m} / 5$, and also $\hat{h}_{\varphi}\left(\varphi^{n}(P)\right)=d^{n} \hat{h}_{\varphi}(P)$, which yields

$$
\varepsilon d^{n} \hat{h}_{\varphi}(P) \leq\left(\frac{\varepsilon}{5} d^{m}\right) \frac{5}{2} d^{n-m} \hat{h}_{\varphi}(P)+c_{9}\left(\hat{h}_{\varphi}(A)+h(\varphi)+1\right) .
$$

A little bit of algebra gives the inequality

$$
\begin{aligned}
n & \leq \log _{d}\left(2 c_{9} \frac{\hat{h}_{\varphi}(A)+h(\varphi)+1}{\varepsilon \hat{h}_{\varphi}(P)}\right) \\
& \leq c_{10}+\log _{d}^{+}\left(\frac{\hat{h}_{\varphi}(A)+h(\varphi)}{\hat{h}_{\varphi}(P)}\right) .
\end{aligned}
$$

Combining the estimate for \# $T_{1}$ with the bounds (25) and (26) for the largest elements in $T_{2}$ and $T_{3}$ completes the proof of (a).

We note that (b) follows immediately from (a).

Finally, we prove (c). Our first observation is that the set $\Upsilon=z\left(\varphi^{-m}(A)\right)$ used in the application of Roth's theorem does not depend on the point $P$. So the largest element in the finite set $T_{1}$ is bounded independently of $P$. (Of course, since Roth's theorem is not effective, we do not have an explicit bound for max $\Upsilon$ in terms $K, S, \varepsilon, \varphi$ and $A$, but that is not relevant.)

Our second observation is to note that the quantity

$$
\hat{h}_{\varphi, K}^{\min } \stackrel{\text { def }}{=} \inf \left\{\hat{h}_{\varphi}(P): P \in \mathbb{P}^{1}(K) \text { wandering for } \varphi\right\}
$$

is strictly positive. To see this, let $P_{0} \in \mathbb{P}^{1}(K)$ be any $\varphi$-wandering point. Then

$$
\hat{h}_{\varphi, K}^{\min }=\inf \left\{\hat{h}_{\varphi}(P): P \in \mathbb{P}^{1}(K) \text { and } 0<\hat{h}_{\varphi}(P) \leq \hat{h}_{\varphi}\left(P_{0}\right)\right\} .
$$

This last set is finite, so the infimum is over a finite set of positive numbers, and hence is strictly positive. Therefore in the upper bounds (25) and (26) for max $T_{2}$ and $\max T_{3}$, we may replace $\hat{h}_{\varphi}(P)$ with $\hat{h}_{\varphi, K}^{\min }$ to get upper bounds independent of $P$. This proves that $\max \left(T_{1} \cup T_{2} \cup T_{3}\right)$ may be bounded independently of $P$, which completes the proof of (c). 


\section{A bound for the number of integral points in an orbit}

In this section, we use Theorem 11 to give a uniform upper bound for the number of $S$-integral points in an orbit.

Corollary 17. Let $K$ be a number field, let $S \subset M_{K}$ be a finite set of places that includes all archimedean places, let $R_{S}$ be the ring of $S$-integers of $K$, and let $d \geq 2$. There is a constant $\gamma=\gamma(d,[K: \mathbb{Q}])$ such that for all rational maps $\varphi \in K(z)$ of degree $d$ satisfying $\varphi^{2}(z) \notin K[z]$ and all $\varphi$-wandering points $P \in \mathbb{P}^{1}(K)$, the number of $S$-integral points in the orbit of $P$ is bounded by

$$
\#\left\{n \geq 1: z\left(\varphi^{n}(P)\right) \in R_{S}\right\} \leq 4^{\# S} \gamma+\log _{d}^{+}\left(\frac{h(\varphi)}{\hat{h}_{\varphi}(P)}\right) .
$$

Proof. By definition, an element $\alpha \in K$ is in $R_{S}$ if and only if $|\alpha|_{v} \leq 1$ for all $v \notin S$, or equivalently, if and only if

$$
h(\alpha)=\sum_{v \in S} d_{v} \log \max \left\{|\alpha|_{v}, 1\right\} .
$$

We note that for $v \in M_{K}^{0}$ we have

$$
\lambda_{v}(\alpha, \infty)=\lambda_{v}([\alpha, 1],[1,0])=\log \max \left\{|\alpha|_{v}, 1\right\} .
$$

The formula for $\lambda_{v}$ when $v$ is archimedean is slightly different, but the trivial inequality $\max \{t, 1\} \leq \sqrt{t^{2}+1}$ shows that for $v \in M_{K}^{\infty}$ we have

$$
\log \max \left\{|\alpha|_{v}, 1\right\} \leq \lambda_{v}(\alpha, \infty)
$$

Hence $\alpha \in R_{S}$ implies $h(\alpha) \leq \sum_{v \in S} d_{v} \lambda_{v}(\alpha, \infty)$.

Let $n \geq 1$ satisfy $z\left(\varphi^{n}(P)\right) \in R_{S}$. Then

$$
h\left(\varphi^{n}(P)\right) \leq \sum_{v \in S} d_{v} \lambda_{v}\left(\varphi^{n}(P), \infty\right) .
$$

Proposition 6 tells us that

$$
h\left(\varphi^{n}(P)\right) \geq \hat{h}_{\varphi}\left(\varphi^{n}(P)\right)-c_{3} h(\varphi)-c_{4}=d^{n} \hat{h}_{\varphi}(P)-c_{3} h(\varphi)-c_{4},
$$

where $c_{3}$ and $c_{4}$ depend only on $d$. Combining (27) and (28) gives

$$
\sum_{v \in S} d_{v} \lambda_{v}\left(\varphi^{n}(P), \infty\right) \geq d^{n} \hat{h}_{\varphi}(P)-c_{3} h(\varphi)-c_{4}
$$

We consider two cases. First, if

$$
d^{n} \hat{h}_{\varphi}(P) \leq 2 c_{3} h(\varphi)+2 c_{4},
$$


then the number of possible values of $n$ is at most

$$
\log _{d}^{+}\left(\frac{2 c_{3} h(\varphi)+2 c_{4}}{\hat{h}_{\varphi}(P)}\right)
$$

which has the desired form. Second, if

$$
d^{n} \hat{h}_{\varphi}(P) \geq 2 c_{3} h(\varphi)+2 c_{4},
$$

then (29) implies that

$$
\sum_{v \in S} d_{v} \lambda_{v}\left(\varphi^{n}(P), \infty\right) \geq \frac{1}{2} d^{n} \hat{h}_{\varphi}(P)=\frac{1}{2} \hat{h}_{\varphi}\left(\varphi^{n}(P)\right) .
$$

Now Theorem 11(b) with $\varepsilon=1 / 2$ and $A=\infty$ tells us that the number of $n$ satisfying (30) is at most

$$
4^{\# S} \gamma_{3}+\log _{d}^{+}\left(\frac{h(\varphi)+\hat{h}_{\varphi}(\infty)}{\hat{h}_{\varphi}(P)}\right),
$$

where $\gamma_{3}$ depends only on $[K: \mathbb{Q}]$ and $d$. (Note that our assumption that $\varphi^{2}(z)$ is not a polynomial is equivalent to the assertion that $\infty$ is not an exceptional point for $\varphi$. This is needed in order to apply Theorem 11.) It only remains to observe that

$$
\hat{h}_{\varphi}(\infty) \leq h(\infty)+c_{3} h(\varphi)+c_{4} \quad \text { and } \quad h(\infty)=h([0,1])=0
$$

to see that the bound (31) has the desired form.

\section{Acknowledgement}

Hsia would like to thank his coauthor and the Department of Mathematics at Brown University for their hospitality during his visit when this work was initiated, and NCTS for his visit during spring 2009. Silverman would like to thank Microsoft Research New England for inviting him to be a visiting researcher during fall 2009.

\section{References}

[Bang 1886] A. S. Bang, “Taltheoretiske Undersogelser”, Tidsskrift Mat. 4:5 (1886), 70-80, 130137. JFM 19.0168 .02

[Gross 1990] R. Gross, “A note on Roth's theorem”, J. Number Theory 36:1 (1990), 127-132. MR 91i:11076 Zbl 0722.11034

[Gross and Silverman 1995] R. Gross and J. Silverman, "S-integer points on elliptic curves", Pacific J. Math. 167:2 (1995), 263-288. MR 96c:11057 Zbl 0824.11038

[Hindry and Silverman 2000] M. Hindry and J. H. Silverman, Diophantine geometry: An introduction, Graduate Texts in Math. 201, Springer, New York, 2000. MR 2001e:11058 Zbl 0948.11023 
[Ingram and Silverman 2009] P. Ingram and J. H. Silverman, "Primitive divisors in arithmetic dynamics", Math. Proc. Cambridge Philosophical Soc. 146:2 (2009), 289-302. MR 2010a:11023 Zbl 05532375

[Lang 1983] S. Lang, Fundamentals of Diophantine geometry, Springer, New York, 1983. MR 85j: 11005 Zbl 0528.14013

[Silverman 1987a] J. H. Silverman, "Arithmetic distance functions and height functions in Diophantine geometry”, Math. Ann. 279:2 (1987), 193-216. MR 89a:11066 Zbl 0607.14013

[Silverman 1987b] J. H. Silverman, “A quantitative version of Siegel's theorem: Integral points on elliptic curves and Catalan curves", J. Reine Angew. Math. 378 (1987), 60-100. MR 89g:11047 Zbl 0608.14021

[Silverman 1993] J. H. Silverman, "Integer points, Diophantine approximation, and iteration of rational maps”, Duke Math. J. 71:3 (1993), 793-829. MR 95e:11070 Zbl 0811.11052

[Silverman 2007] J. H. Silverman, The arithmetic of dynamical systems, Graduate Texts in Math. 241, Springer, New York, 2007. MR 2008c:11002 Zbl 1130.37001

[Silverman 2009] J. H. Silverman, "Height estimates for equidimensional dominant rational maps", preprint, 2009. To appear in J. Ramanujan Math. Soc. arXiv 0908.3835

[Silverman and Voloch 2009] J. H. Silverman and J. F. Voloch, "A local-global criterion for dynamics on $\mathbb{P}^{1} "$, Acta Arith. 137:3 (2009), 285-294. MR 2010g:37173 Zbl 05538712

[Zsigmondy 1892] K. Zsigmondy, "Zur Theorie der Potenzreste”, Monatsh. Math. Phys. 3:1 (1892), 265-284. MR 1546236 JFM 24.0176.02

Received October 22, 2009.

LIANG-CHUNG HSIA

DEPARTMENT OF MATHEMATICS

NATIONAL CEnTRAL University

CHUNG-Li 32054

TAIWAN

hsia@math.ncu.edu.tw

JOSEPH H. SILVERMAN

Department of Mathematics, Box 1917

BROWN UNIVERSITY

151 THAYER STREET

PROVIDENCE, RI 02912

UNITED STATES

jhs@math.brown.edu

http://www.math.brown.edu/ jhs 


\title{
PACIFIC JOURNAL OF MATHEMATICS
}

\author{
http://www.pjmath.org \\ Founded in 1951 by
}

E. F. Beckenbach (1906-1982) and F. Wolf (1904-1989)

\section{EDITORS}

V. S. Varadarajan (Managing Editor)

Department of Mathematics

University of California

Los Angeles, CA 90095-1555

pacific@math.ucla.edu

Vyjayanthi Chari

Department of Mathematics

University of California

Riverside, CA 92521-0135

chari@math.ucr.edu

\section{Robert Finn}

Department of Mathematics Stanford University

Stanford, CA 94305-2125

finn@math.stanford.edu

Kefeng Liu

Department of Mathematics

University of California

Los Angeles, CA 90095-1555

liu@math.ucla.edu
Darren Long

Department of Mathematics

University of California

Santa Barbara, CA 93106-3080

long@math.ucsb.edu

Jiang-Hua Lu

Department of Mathematics

The University of Hong Kong

Pokfulam Rd., Hong Kong jhlu@maths.hku.hk

Alexander Merkurjev

Department of Mathematics University of California

Los Angeles, CA 90095-1555 merkurev@math.ucla.edu
Sorin Popa

Department of Mathematics

University of California

Los Angeles, CA 90095-1555

popa@math.ucla.edu

Jie Qing

Department of Mathematics

University of California

Santa Cruz, CA 95064

qing@ cats.ucsc.edu

Jonathan Rogawski

Department of Mathematics

University of California

Los Angeles, CA 90095-1555

jonr@math.ucla.edu

\section{PRODUCTION}

pacific@math.berkeley.edu

Silvio Levy, Scientific Editor Matthew Cargo, Senior Production Editor

ACADEMIA SINICA, TAIPEI

CALIFORNIA INST. OF TECHNOLOGY

INST. DE MATEMÁTICA PURA E APLICADA

KEIO UNIVERSITY

MATH. SCIENCES RESEARCH INSTITUTE

NEW MEXICO STATE UNIV.

OREGON STATE UNIV.

\section{SUPPORTING INSTITUTIONS}

STANFORD UNIVERSITY
UNIV. OF BRITISH COLUMBIA
UNIV. OF CALIFORNIA, BERKELEY
UNIV. OF CALIFORNIA, DAVIS
UNIV. OF CALIFORNIA, LOS ANGELES
UNIV. OF CALIFORNIA, RIVERSIDE
UNIV. OF CALIFORNIA, SAN DIEGO
UNIV. OF CALIF., SANTA BARBARA

UNIV. OF CALIF., SANTA CRUZ

UNIV. OF MONTANA

UNIV. OF OREGON

UNIV. OF SOUTHERN CALIFORNIA

UNIV. OF UTAH

UNIV. OF WASHINGTON

WASHINGTON STATE UNIVERSITY

These supporting institutions contribute to the cost of publication of this Journal, but they are not owners or publishers and have no responsibility for its contents or policies.

See inside back cover or www.pjmath.org for submission instructions.

The subscription price for 2011 is US \$420/year for the electronic version, and \$485/year for print and electronic.

Subscriptions, requests for back issues from the last three years and changes of subscribers address should be sent to Pacific Journal of Mathematics, P.O. Box 4163, Berkeley, CA 94704-0163, U.S.A. Prior back issues are obtainable from Periodicals Service Company, 11 Main Street, Germantown, NY 12526-5635. The Pacific Journal of Mathematics is indexed by Mathematical Reviews, Zentralblatt MATH, PASCAL CNRS Index, Referativnyi Zhurnal, Current Mathematical Publications and the Science Citation Index.

The Pacific Journal of Mathematics (ISSN 0030-8730) at the University of California, c/o Department of Mathematics, 969 Evans Hall, Berkeley, CA 94720-3840, is published monthly except July and August. Periodical rate postage paid at Berkeley, CA 94704, and additional mailing offices. POSTMASTER: send address changes to Pacific Journal of Mathematics, P.O. Box 4163, Berkeley, CA 94704-0163.

PJM peer review and production are managed by EditFLOW ${ }^{\mathrm{TM}}$ from Mathematical Sciences Publishers.

PUBLISHED BY PACIFIC JOURNAL OF MATHEMATICS

at the University of California, Berkeley 94720-3840

A NON-PROFIT CORPORATION

Typeset in IATEX

Copyright $(2011$ by Pacific Journal of Mathematics 


\section{PACIFIC JOURNAL OF MATHEMATICS}

Volume $249 \quad$ No. $2 \quad$ February 2011

A gluing construction for prescribed mean curvature

257

ADRIAN BUTSCHER

Large eigenvalues and concentration

271

BRUNO COLBOIS and ALESSANDRO SAVO

Sur les conditions d'existence des faisceaux semi-stables sur les courbes multiples primitives

JEAN-MARC DRÉZET

A quantitative estimate for quasiintegral points in orbits

LIANG-CHUNG HSIA and JosePh H. Silverman

Möbius isoparametric hypersurfaces with three distinct principal curvatures, II

ZEJUN HU and SHUJIE ZHAI

Discrete Morse theory and Hopf bundles

371

DMITRY N. KOZLOV

Regularity of canonical and deficiency modules for monomial ideals

ManOJ KUMmini and SATOSHI MURAI

$\mathrm{SL}_{2}(\mathbb{C})$-character variety of a hyperbolic link and regulator

WEIPING LI and QINGXUE WANG

Hypergeometric evaluation identities and supercongruences

LING LONG

Necessary and sufficient conditions for unit graphs to be Hamiltonian

H. R. Maimani, M. R. Pournaki and S. Yassemi

Instability of the geodesic flow for the energy functional

DOMENico PERrone

String structures and canonical 3-forms

CORBETT REDDEN

Dual pairs and contragredients of irreducible representations

BINYONG SUN

On the number of pairs of positive integers $x_{1}, x_{2} \leq H$ such that $x_{1} x_{2}$ is a $k$-th power

DOYCHIN I. TOLEV

Correction to the article A Floer homology for exact contact embeddings 